\title{
Message from the President-Elect of the World Federation of Nuclear Medicine and Biology
}

\author{
Dong Soo Lee ${ }^{1,2}$
}

Published online: 13 November 2017

(C) Korean Society of Nuclear Medicine 2017

The 12th Asia Oceania Congress of Nuclear Medicine has just come to an end. Once again this congress corroborated the fact that in Asia and the Oceania region, though many efforts have so far been delivered by the leaders, nuclear medicine is in its infancy in Asia. In the eloquent era of global precision and personalized medicine, most Asian countries are still struggling with the infrastructure of nuclear medicine. Exceptions are Japan, Korea, and China. They preform 5 million nuclear medicine procedures (imaging and therapy) annually: 2 million in Japan, 1 million in Korea, and 2 million in China. As expected, each country has its own problems or hurdles to overcome, i.e., Bangladesh has well-designed infrastructure but economic development has not caught up; Mongolia, Yemen, and Nepal have imminent work to raise pioneers of nuclear medicine physicians at home; China needs to immediately educate a multitude of nuclear medicine physicians and scientists to double the institution and clinical volumes in every prefecture. Japan has witnessed the decline of nuclear medicine practices recently and the loss of nuclear medicine's disciplinary standing while securing subspecialty status according to the recent progress of legalization of disciplines. Korea has experienced a painful setback, to the level of 10 years ago, in the clinical volume of PET procedures due to an irrational amendment of PET reimbursement guidelines

The author is Editor-in-Chief of this journal and President-Elect of the World Federation of Nuclear Medicine and Biology (WFNMB).

Dong Soo Lee dsl@snu.ac.kr

1 Nuclear Medicine, Seoul National University (SNU) and SNU Hospital, Seoul, South Korea

2 Molecular Medicine and Biopharmaceutical Sciences, SNU, Seoul, South Korea in the national health insurance system. All of these challenges and changes have existed since the birth of our discipline in every country as well as globally. The responses to these challenges damaged and in some cases strengthened our discipline's status directing our endeavor to make nuclear medicine more appropriate and beneficial to clinicians and patients.

What we need currently from the global perspective is to share our success in overcoming these challenges and strategic plans to overcome the approaching future problems. It is fortunate that the current leadership of the World Federation of Nuclear Medicine and Biology (WFNMB) have nearly concreted the relationship with the World Health Organization (WHO). They submitted the application for approval by the WHO to recognize the WFNMB as an official NonGovernmental Organization (NGO) at the international level. The recognition of the WFNMB by the WHO means that the WFNMB is considered an official partner and more importantly that nuclear medicine is considered a clinical entity. This recognition process is long overdue. Once the approval is done, we will need to construct our relationship with regional offices. Uniquely, the WHO works at the regional or country level where the working plans are devised and the budget is spent mostly by regional offices (ROs). Six regional offices of the WHO work globally and, for example, the Asian region is covered by three regional offices, i.e., Western Pacific RO (WPRO), South Eastern Asian RO (SEARO), and Eastern Mediterranean RO (EMRO). Once the WHO annouces nuclear medicine as helpful for the maintenance of human health, nuclear medicine or WFNMB shall join the efforts to achieve the Sustainability Development Goal 3 (SDG) among the United Nations-set 17 SDGs. Under the WFNMB and WHO umbrella, the Asia Regional Cooperative Council for Nuclear Medicine (ARCCNM) and Arabic Society of Nuclear Medicine shall begin to collaborate with WPRO, SEARO, and EMRO. 
To assure the contribution of nuclear medicine to the improvement of global human health, we need to be part of the Integrated Service Delivery by the WHO and join the effort to solve the problem of a needs-based shortage of health workers in developing countries. As expected, the secondary care parts of nuclear medicine are therapy and theranostics. Cuttingedge PET/MRI or PET/CT are beyond secondary care and instead have radionuclide therapy and dosimetry as the essential part. Fortunately, the introduction of this therapy/ theranostics does not require much investment, but we need to train radiopharmacists, nuclear medicine physicists, and nuclear medicine physicians. SDG3 announced that premature mortality of non-communicable disease should be decreased by one third by 2030 and nuclear medicine can help directly increase survival of cancer patients and indirectly the longevity and further quality of life of heart and brain disease patients. We can even use nuclear medicine in the secondary prevention of many non-communicable diseases although it might not join the works of primary prevention in the aging obese population of developing countries. The International Atomic Energy Agency will booster the infrastructure of both facility and human resources and supply the know-hows to safely run this infrastructure, and the WHO will ask us, the nuclear medicine experts either physicians or scientists, to respond to the global, regional, and country-level health problems. Interestingly, only one fifth of the WHO efforts and budget are said to be spent to control global, grave infectious diseases and the remaining four fifths are used for other global health problems, such as environmental health, occupational health, fine dust problems affecting public health, and other common community health problems. As a recognized discipline from the viewpoint of health defined by the WHO, we can overcome the shortcomings of our discipline, which had been considered to be more destined to benefit people who can afford it at an individual or country level. In developing countries of the world, many people are still waiting for the benefit of nuclear medicine.

To meet the needs of people at the global level, do we need to invest in costly instruments in developing countries? I personally believe that the investment of this basic infrastructure is the responsibility of each country. What we need rather is to innovate the therapy/theranostics of our nuclear medicine discipline to meet the unmet clinical needs, and all the other endeavors of molecular imaging, physics and dosimetry, radiochemistry, and radionanomedicine should serve this goal. The WFNMB shall further represent the needs of developing countries since though there have been great SNMMI and EANM for many decades to lead and innovate our discipline they usually only suffice to help their own people. The WFNMB along with these advanced workforces will seek breakthroughs in both research and clinical practice, especially for secondary care and novel theranostics. Innovative progress in theranostics can be achieved by the best nuclear medicine people from both developing and developed counties. The Theranostics World Congress 2019 at Jeju Island shall be a good opportunity to seek the future direction of developing countries. There are many chances to become firstmovers. Maintaining or establishing an independent law- or government-endorsed discipline of nuclear medicine is the prerequisite in each country and recruitment of the best scientific minds among physicists and chemists/pharmacists to nuclear medicine is the next urgent endeavor. Finally, devising a totally new instrument enabling almost a negligent amount of radioactivity of radiopharmaceuticals would also be greatly appreciated. For the last entity, during the AOCNMB congress in the first week of October, American physicists and Chinese industry promised the release of a whole body PET/CT. It was really invigorating with expected patient radiation of $0.5 \mathrm{mSv}$ per study, which is at least one tenth of the current PET/CT or PET/MRI level. The best science accompanied by needsbased theranostics as the primary priority is the future of our field. We've had a long journey from hybrid imaging and the invention of small animal-dedicated instruments while researching even clinically-non-translatable modalities of molecular imaging. Let us do our sincere part to see positive results, especially in the less/least developed countires of the world.

The current risk of nuclear medicine is not going to be tamed by the advancement of nuclear medicine in the developed countries alone but by the shared advancement in developing countries. Survival depends upon the success of sharing and geographic expanding, not the prosperity of only part of the world, such as the USA or western part of the European Union. 